\title{
Degradation of Iodinated Contrast Media in Aquatic Environment by Means of UV, UV/TiO 2 Process, and by Activated Sludge
}

\author{
Ewa Borowska • Ewa Felis • Sebastian Żabczyński
}

Received: 6 November 2014 / Accepted: 11 March 2015 / Published online: 17 April 2015

(C) The Author(s) 2015. This article is published with open access at Springerlink.com

\begin{abstract}
Iodinated contrast media (ICM), which are used for radiological visualization of human tissue and cardiovascular system, are poorly biodegradable; hence, new methods of their removal are sought. In this study, the effectiveness of selected X-ray ICM removal by means of $\mathrm{UV}$ and $\mathrm{UV} / \mathrm{TiO}_{2}$ pretreatment processes from synthetic hospital wastewater was demonstrated. The following compounds were investigated: iodipamide, iohexol, and diatrizoate. The experiments were as follows: (i) estimated susceptibility of the ICM to decay by UV radiation in different aquatic matrices, (ii) determined an optimal retention time of hospital wastewater in the UV reactor, (iii) determined optimum $\mathrm{TiO}_{2}$ concentration to improve the effectiveness of the UV pretreatment, and (iv) investigated removal of ICM by combination of the photochemical and biological treatment methods. The quantum yields of selected ICM decay in deionized water $(\mathrm{pH}=7.0)$ were established as $0.006,0.004$, and 0.029 for iohexol, diatrizoate, and iodipamide, respectively. Furthermore, the experiments revealed that diatrizoate and iohexol removal in the UV/ $\mathrm{TiO}_{2}$ process is more efficient than in UV process alone. For diatrizoate, the removal efficiency equaled to 40 and
\end{abstract}

E. Borowska $\cdot$ E. Felis $\cdot$ S. Żabczyński

Environmental Biotechnology Department, The Silesian

University of Technology, Akademicka 2,

44-100 Gliwice, Poland

E. Borowska $\cdot$ E. Felis $(\bowtie)$

Centre for Biotechnology, The Silesian University of

Technology, Krzywoustego 8, 44-100 Gliwice, Poland

e-mail: ewa.felis@polsl.pl
$30 \%$, respectively, and for iohexol, the efficiency was 38 and $27 \%$, respectively. No significant increase in iodipamide removal in $\mathrm{UV}$ and $\mathrm{UV} / \mathrm{TiO}_{2}$ processes was observed (29 and $28 \%$, respectively). However, highest removal efficiency was demonstrated in synthetic hospital wastewater with the combined photochemical and biological treatment method. The removal of diatrizoate and iohexol increased to at least $90 \%$, and for iodipamide, to at least $50 \%$.

Keywords Iodinated contrast media Membrane bioreactors Photolysis $\cdot \mathrm{UV} / \mathrm{TiO}_{2}$ process

$\begin{array}{ll}\text { Abbreviations } \\ \text { CAS } & \text { Chemical abstracts service } \\ \text { COD } & \text { Chemical oxygen demand } \\ \text { ICM } & \text { Iodinated contrast media } \\ \text { HPLC } & \text { High-pressure liquid chromatography } \\ \text { LOQ } & \text { Limit of quantification } \\ \text { MBR } & \text { Membrane bioreactor } \\ \text { SBR } & \text { Sequencing batch reactor } \\ \text { UV } & \text { Ultraviolet } \\ \text { WWTP } & \text { Wastewater treatment plant }\end{array}$

\section{Introduction}

The occurrence and ecotoxicological effects of the pharmaceuticals in the aquatic environment have been defined as one of the most emerging problems in the environmental chemistry (Daughton and Ternes 1999; Halling-Sorensen et al. 1998; Heberer 2002; Kummerer 
2001, 2009). More than 80 pharmaceutically active compounds, including iodinated X-ray contrast media, have been detected up to the microgram per liter levels in sewage, surface, and ground waters (Heberer 2002; Mompelat et al. 2009). Iodinated X-ray contrast media (ICM) are frequently applied in clinical diagnosis for imaging soft tissues such as blood vessels and organs (Christiansen 2005). The structure of ICM is based on a benzene ring containing three iodine substituents which increase X-ray absorption and thereby allow for visualization of the organ or tissue. Furthermore, these polar atoms ensure high water solubility. However, their chemical structure is very stable in human organisms, and thus, ICM are excreted via urine mostly in unmetabolized form (Seitz et al. 2006). Taking in account the high numbers of X-ray examinations performed each year, this results in high volumes of ICM compounds released into the environment. For example, in Germany alone, approximately $500 \mathrm{t}^{\text {annum }}{ }^{-1}$ of ICM are applied (Schulz et al. 2008), with hospital wastewater as the main source of ICM (Knodel et al. 2011).

It has been shown that ICM could not be eliminated completely by the conventional wastewater treatment processes, which results in their discharge into the aquatic environment (Perez and Barcelo 2007; Putschew et al. 2000; Ternes and Hirsch 2000). The ICM has been detected in many types of aquatic matrices. For example, in Germany, the concentration levels of diatrizoate, iopromide, and iomeprol frequently exceeded $1 \mu \mathrm{g} \mathrm{L}^{-1}$ in the influent and the effluent of a municipal wastewater treatment plant (WWTP) (Ternes and Hirsch 2000).

Putschew et al. (2000) detected ICM in all water bodies, i.e., WWTP influents and effluents, surface water, as well as in bank filtrate and drinking water. Thus, in all countries with developed medical care system, ICM are expected to be present at appreciable quantities in sewage effluence and eventually in receiving waters. Their stable chemical structure results in persistence in the environment (Heberer 2002). Few ecotoxicological studies on toxicity of selected ICM showed that their release into wastewater and surface water is not expected to pose a threat to the aquatic environment (StegerHartmann et al. 1999, 2002; Haiss and Kummerer 2006). Nevertheless, high concentration of ICM in the aquatic environment and their highly persistent nature cannot be ignored. Ubiquitous presence of ICM and their degradation products became a reason for monitoring of these substances in the aquatic environment (Seitz et al. 2006).
In order to minimize the amount of ICM discharged into the aquatic environment, new methods of their degradation are investigated. Apart from biological methods, chemical techniques seem to be an attractive option. In particular, photochemical processes seem suitable due to their high effectiveness in pharmaceuticals removal from wastewater (Klavarioti et al. 2009). Among these methods, ICM degradation was already investigated in the advanced oxidation processes (involving reactive species) such as $\mathrm{UV} / \mathrm{H}_{2} \mathrm{O}_{2}, \mathrm{UV} / \mathrm{TiO}_{2}$, and $\mathrm{O}_{3} / \mathrm{H}_{2} \mathrm{O}_{2}$ (Doll and Frimmel 2004; Huber et al. 2005; Ternes et al. 2003). Doll and Frimmel (2003) investigated photolysis of ICM by stimulated solar UV radiation. Ternes et al. (2003) compared the efficiency of the ozonolysis and advanced oxidation processes $\left(\mathrm{O}_{3} /\right.$ UV-low pressure mercury lamp, $\mathrm{O}_{3} / \mathrm{H}_{2} \mathrm{O}_{2}$ ) for ICM removal from municipal wastewater. However, in their study, usage of ozone in the concentration range of 10 $15 \mathrm{mg} \mathrm{L}^{-1}$ did not result in complete ICM removal, and the advanced oxidation processes mentioned above did not cause significantly higher ICM removal than ozone alone. Huber et al. (2005) also concluded that ozonation itself was insufficient for complete elimination of ICM from wastewater. Ning et al. (2009) combined ozone with ultrasound irradiation treatment and reported almost complete decay of selected X-ray contrast media, while Kwon et al. (2012) evaluated an effective removal of iopromide from municipal wastewater using electron beam irradiation technology.

In this study, a hybrid treatment was investigated, which combined the chemical (i.e., photochemical) and conventional biological treatment processes. With this approach, chemical oxidation was used in order to disrupt the original structure of the ICM pollutant in order to make its biodegradation more feasible (Miksch et al. 2015). The aim of our study was therefore to examine the efficiency of ICM removal from synthetic hospital wastewater by $\mathrm{UV}$ and $\mathrm{UV} / \mathrm{TiO}_{2}$ processes and in combination with biological treatment in membrane bioreactors (MBRs). The work was divided into four tasks: (i) estimation of the susceptibility of selected ICM to decay by UV radiation in different aquatic matrices, (ii) determination of optimum hospital wastewater retention time in the UV reactor, (iii) determination of optimal $\mathrm{TiO}_{2}$ concentration for the improvement of the effectiveness of UV treatment, and (iv) comparison of the UV and UV/ $\mathrm{TiO}_{2}$ process effectiveness in the hybrid installation. Three representatives of ICM were selected for the experiments: diatrizoate, iohexol, and iodipamide. 


\section{Materials and Methods}

\subsection{Chemical Standards}

Analytical standards of iohexol, iodipamide, and diatrizoate were purchased from Sigma-Aldrich. The characteristic feature of ICM structure is at least one benzene ring substituted with iodine atoms. Additionally, the aromatic rings are substituted by alkyl side chains coupled to the aromatic ring through amide linkages. The presence of hydroxyl groups improves water solubility of the compounds. In regard to structure, the selected ICM represent to groups: iodipamide and diatrizoate are considered as ionic contrast media, while iohexol is considered as a nonionic contrast medium (see Table 1).

Tetrabutylammonium bromide (TBAB) was purchased from Fluka. Titanium oxide (CAS number 1317-70-0) was purchased from Sigma-Aldrich. During the investigation, a powdered anatase form of $\mathrm{TiO}_{2}$ (metals basis $\approx 99.8 \%$ ) was used.

\subsection{Detection of ICM by HPLC}

The ICM concentrations were determined by highperformance liquid chromatography (HPLC UltiMate3000, Dionex). Analysis of iodipamide concentration was performed on Hypersil ${ }^{\mathrm{TM}}$ GOLD column (Thermo Scientific). Flow rate was set at $0.3 \mathrm{~mL} \mathrm{~min}^{-1}$. The mobile phase was composed of acetate buffer, acetonitryle, and Milli-Q Water in volumetric ratio of 3:42:55. The detection of iodipamide was performed at the wavelength of $238 \mathrm{~nm}$. Analysis of diatrizoate and iohexol concentrations was performed on $\mathrm{C} 8$ column (Merck). Flow rate was set at $1.1 \mathrm{~mL} \mathrm{~min}^{-1}$. The mobile phase was composed of phosphate buffer and methanol containing TBAB in volumetric ratio of $85: 15$. The diatrizoate detection was at the wavelength of $238 \mathrm{~nm}$ and the iohexol at the wavelength of $265 \mathrm{~nm}$. The limit of quantification (LOQ), in the case of all the investigated compounds, was equal to $0.2 \mathrm{mg} \mathrm{L}^{-1}$. It was established as the second lowest calibration point of their calibration curves (linear regression, $R^{2}>0.98$ ), and the calculated "signal to noise" ratio $(\mathrm{S} / \mathrm{N})$ of the compounds was greater than 10. The limits of detection (LODs) of the investigated ICMs were defined when $\mathrm{S} / \mathrm{N}$ was at the level of 3. LODs in all cases were equal to $0.05 \mathrm{mg} \mathrm{L}^{-1}$. The accuracies of the methods were calculated according to ISO 17025, as a recovery. The calculated values of the recoveries were equal to $116.5,104.4$, and $92.4 \%$ for iohexol, diatrizoate, and iodipamide, respectively.

\section{$2.3 \mathrm{UV}$ and $\mathrm{UV} / \mathrm{TiO}_{2}$ Setup}

The UV and $\mathrm{UV} / \mathrm{TiO}_{2}$ processes were performed in the UV glass reactor of 350-mL volume, equipped with a polychromatic medium-pressure mercury lamp, with emission spectra from 255 to $579 \mathrm{~nm}$ (UVI LabP400, Vita Tech, Germany). The lamp emission spectrum was measured by means of Ocean Optic radiophotometer (Fig. 1). The average path length of light through the solution was equal to $10 \mathrm{~mm}$. The light source was characterized with uranyl oxalate as an actinometer according to Miller and Olejnik (2001). The nominal power of UV lamp was equal to $200 \mathrm{~W}$. In the photochemical process experiments, the UV reactor was operated either in a recirculation loop (experiments in Sects. 3.1, 3.2, and 3.3) or as a plug-flow reactor (experiments in Sect. 3.4). The detailed description and the schematic diagram of the UV reactor operated in various flow modes is described in our previous publication (Felis et al. 2009).

\subsection{Deionized and Tap Water Experiments}

The water composition can affect the photochemical reaction rate, especially the presence of inorganic ions (e.g., bicarbonate and carbonate ions). In order to determine the matrix effect on the photochemical decay of selected ICM, the investigations were conducted in both deionized (Milli-Q Water, Millipore) and tap water (all results in Sect. 3.1). The experiments were performed in water spiked with the analytical standards of iohexol (10 $\left.\mathrm{mg} \mathrm{L}^{-1}\right)$, diatrizoate $\left(5 \mathrm{mg} \mathrm{L}^{-1}\right)$, and iodipamide $\left(10 \mathrm{mg} \mathrm{L}^{-1}\right)$. The basic information on composition of tap water used in the experiments is shown in Table 2.

\subsection{Synthetic Hospital Wastewater Experiments}

The experiments presented in Sects. 3.2-3.4 were performed in synthetic hospital wastewater (average COD of $\left.1.3 \mathrm{~g} \mathrm{~L}^{-1}\right)$, composed of $\mathrm{NH}_{4} \mathrm{Cl}\left(0.250 \mathrm{~g} \mathrm{~L}^{-1}\right)$, $\mathrm{KH}_{2} \mathrm{PO}_{4}\left(0.044 \mathrm{~g} \mathrm{~L}^{-1}\right)$, and $\mathrm{CH}_{3} \mathrm{COONa}$ $\left(1.670 \mathrm{~g} \mathrm{~L}^{-1}\right)$. This hospital wastewater was enriched by iohexol $\left(1.0 \mathrm{mg} \mathrm{L}^{-1}\right)$, iodipamide $\left(1.5 \mathrm{mg} \mathrm{L}^{-1}\right)$, and diatrizoate $\left(1.0 \mathrm{mg} \mathrm{L}^{-1}\right)$ standards. 
Table 1 Basic data concerning ICMs used in the studies

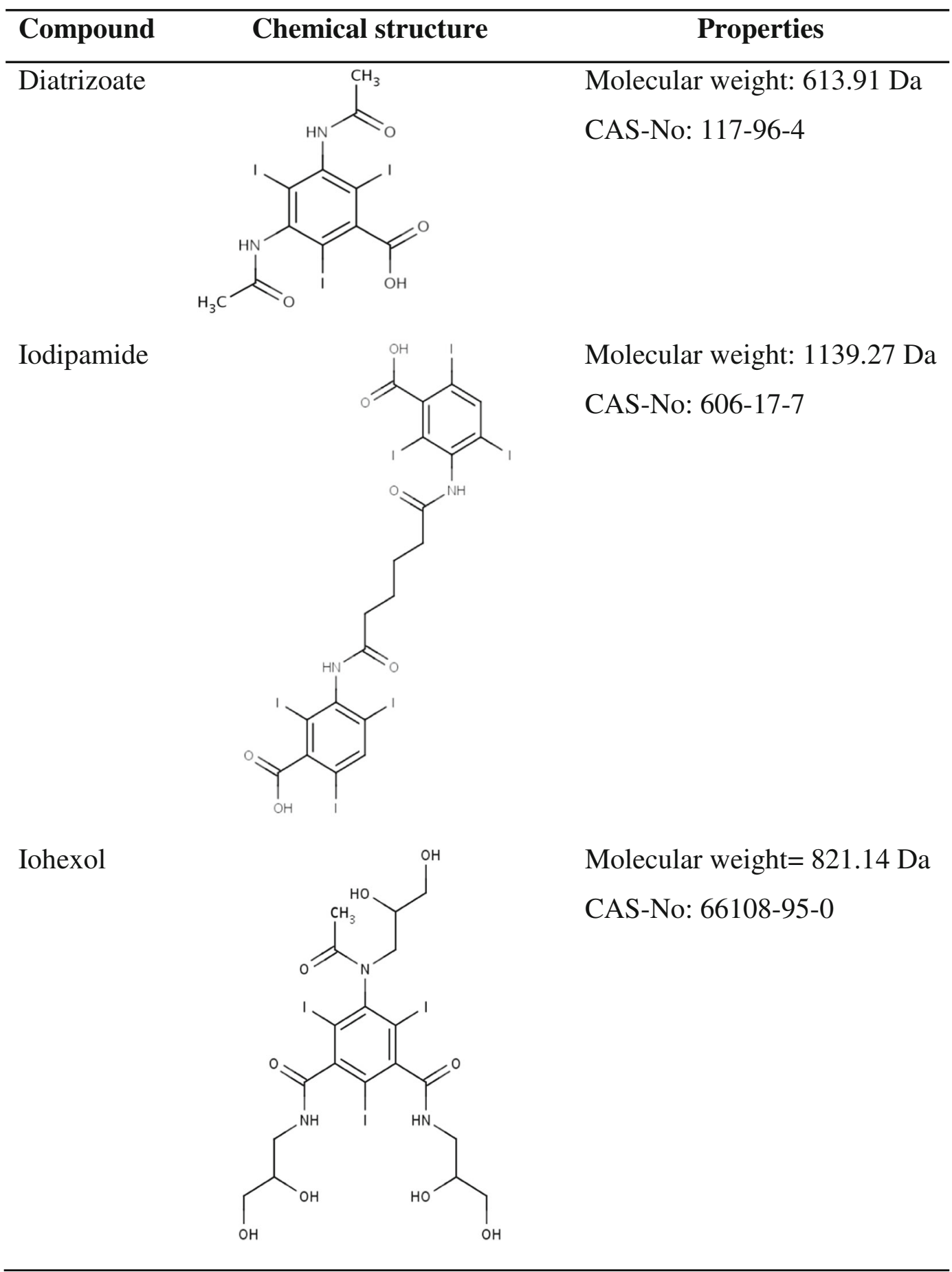

2.6 Membrane Bioreactors Setup

In this part of the study, two MBRs - control MBR and hybrid MBR - were used. The MBRs were equipped with A4 Size Mat Sheet Membrane, Kubota System (0.4- $\mu \mathrm{m}$ pore size). The working volume of both MBRs was equal to $30 \mathrm{~L}$, and the average concentration of activated sludge in the MBRs was at the level of 
Fig. 1 Medium-pressure $\mathrm{Hg}$ lamp emission spectrum with reference to absorbance of investigated ICM (water, $\mathrm{pH}=7.0)$

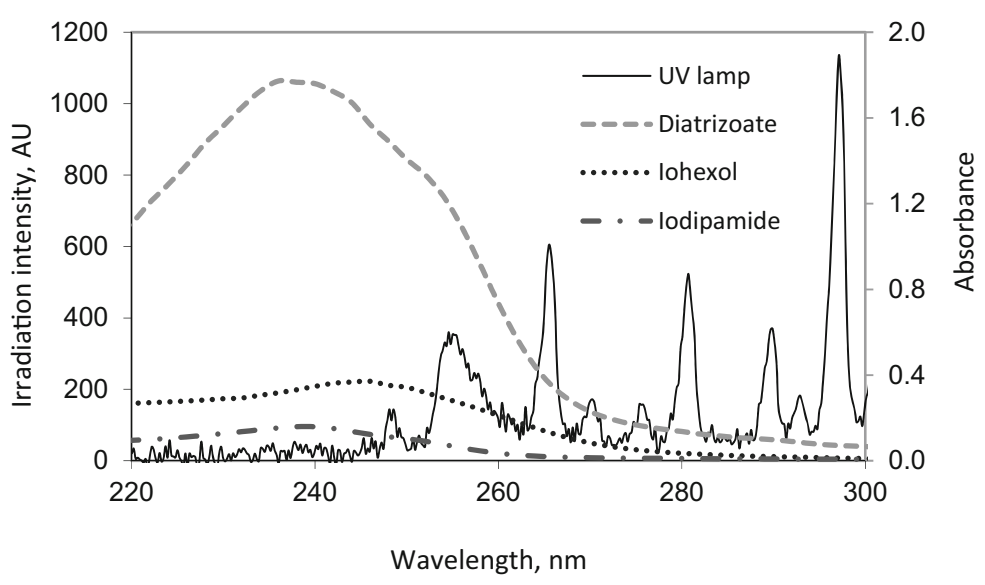

$4.5 \mathrm{~g} \mathrm{~L}^{-1}$. The sludge retention time was equal to 24 days, with hydraulic retention time maintained at $84 \mathrm{~h}$ in both bioreactors. The MBRs were fed with synthetic hospital wastewater, with composition described in Sect. 2.3. The control membrane bioreactor was fed with a synthetic wastewater without photochemical pretreatment. Synthetic hospital wastewater after respectively $\mathrm{UV}$ and $\mathrm{UV} / \mathrm{TiO}_{2}$ treatment (irradiation time $=4 \mathrm{~min}$ ) was introduced as a feeding medium into the hybrid membrane reactor. The experiments were performed after the sludge adaptation period ( 72 days), when the sludge retention time has been stabilized.

\subsection{Quantum Yields}

Kinetic equations were used to quantitatively characterize the UV oxidation. Quantum yields are useful in the design of full-scale installations due to more general

Table 2 Basic parameters of the tap water used in photolytic experiments

\begin{tabular}{llc}
\hline Parameters & Unit & Value \\
\hline $\mathrm{pH}$ & - & 7.4 \\
Thermal conductivity $\left(25^{\circ} \mathrm{C}\right)$ & $\mu \mathrm{S} \mathrm{cm}^{-1}$ & 1118 \\
$\mathrm{NH}_{4}$ & $\mathrm{mg} \mathrm{L}^{-1}$ & $<0.06$ \\
$\mathrm{NO}_{2}$ & $\mathrm{mg} \mathrm{L}^{-1}$ & 4.2 \\
$\mathrm{NO}_{3}$ & $\mathrm{mg} \mathrm{L}^{-1}$ & $<0.03$ \\
$\mathrm{Fe}$ & $\mathrm{mg} \mathrm{L}^{-1}$ & 0.0254 \\
$\mathrm{Mn}$ & $\mathrm{mg} \mathrm{L}^{-1}$ & 0.0159 \\
Total hardness & $\mathrm{mg} \mathrm{CaCO}_{3} \mathrm{~L}^{-1}$ & 458 \\
& $\mathrm{mmol} \mathrm{L}^{-1}$ & 4.58 \\
& ${ }^{\circ} \mathrm{dH}^{2}$ & 25.7 \\
\hline
\end{tabular}

nature of the equations. The reaction rates of the ICM photochemical decay is described by Eq. (1), which is a combination of Stark-Einstein and Lambert-Beer law:

$r_{\mathrm{UV}}=-\frac{d C}{d t}=\phi \times E_{0} \times\left(1-10^{-\varepsilon \times b \times C}\right)$

where $r_{\mathrm{UV}}$ is the initial reaction rate, $C$ is the ICM initial (molar) concentration, $\varphi$ is the quantum yield, $E_{0}$ is the lamp irradiance, $b$ is the average light path into the solution, and $\varepsilon$ is the weighted average molar extinction coefficient.

After a mathematical transformation of Eq. (1), the values of quantum yields of photochemical decay can be calculated according to Eq. (2):

$\phi=\frac{r_{\mathrm{UV}}}{E_{0} \times\left(1-10^{-\varepsilon \times b \times C}\right)}$

The calculations were performed as in the previous study (Felis et al. 2011) - the initial reaction rates $\left(r_{\mathrm{UV}}\right)$ were calculated by differentiating exponential curve that fitted experimental points $(C, t)$ at the correlation factor higher than 0.95 . The radiation from the region of 254 to $313 \mathrm{~nm}$ was used in all performed investigations, which corresponds to a region where the UV lamp emission (254 to $579 \mathrm{~nm}$ ) and the absorption spectrum of ICM (200 to $313 \mathrm{~nm}$ ) overlapped. The acinometric investigations concluded that the lamp irradiance $\left(E_{0}\right)$ was equal to $6.2 \times 10^{-6} \mathrm{E} \mathrm{L}^{-1} \mathrm{~s}^{-1}\left(2.46 \mathrm{~W} \mathrm{~L}^{-1}\right)$. The introduction in kinetic considerations of a weighted average molar absorption coefficient $(\varepsilon)$ allowed to determine the actual participation of each wavelength in the absorption spectra of the studied ICM. The $\varepsilon$ parameter for each ICM was calculated as a weighted average of single molar extinction coefficients determined at selected 
wavelengths ( $\lambda=$ every $2 \mathrm{~nm}$, in the range of active spectrum from 254 to $313 \mathrm{~nm}$ ).

\section{Results and Discussion}

3.1 Photolysis of Selected ICM in Aquatic Solution with Quantum Yield Determination

This part of the study determined the susceptibility of selected ICM (iohexol, iodipamide, and diatrizoate) to degradation by the polychromatic UV radiation, emitted by the medium-pressure $\mathrm{Hg}$ lamp. The absorbance of selected ICM dissolved in water $\left(\mathrm{pH}=7, t \approx 20^{\circ} \mathrm{C}\right)$ with reference to an emission spectra of medium-pressure $\mathrm{Hg}$ lamp is presented in Fig. 1. The experiments were performed in deionized (no ions interference with the UV light) and in tap water.

\subsection{Photolysis in Deionized Water}

The average initial concentrations of ICM during the experiment performed in deionized water were as follows: 11.1, 5.0, and $10.1 \mathrm{mg} \mathrm{L}^{-1}$ for iohexol, diatrizoate, and iodipamide, respectively. Among the studied ICM, iohexol was the least efficiently removed in deionized water in the UV process. After 60 min of irradiation, its concentration in reaction solution was equal to $1.8 \mathrm{mg} \mathrm{L}^{-1}$, which corresponds to removal of $84 \%$. Both diatrizoate and iodipamide were more susceptible to the decay by UV radiation (than iohexol), and after $30 \mathrm{~min}$, their concentrations in the solution were at the limit of quantification $\left(\mathrm{LOQ}=0.2 \mathrm{mg} \mathrm{L}^{-1}\right)$. This corresponded to removal degrees of 96 and $98 \%$ for diatrizoate and iodipamide, respectively. The removal of selected ICM during direct photolysis performed in deionized water is presented in Fig. 2.

\subsection{Quantum Yield Determination}

The data used to calculate the quantum yields were taken from the deionized water experiment and are summarized in Table 3. The quantum yields of selected ICM decay in the aqueous solution $(\mathrm{pH}=7.0)$ were established as 0.006, 0.004, and 0.029 for iohexol, diatrizoate, and iodipamide, respectively. This means that iodipamide was the most susceptible to degradation by direct photolysis (as a consequence of light absorption), followed by diatrizoate and by iohexol. The obtained results strongly indicate which of the chemical compounds are most susceptible to this type of degradation; however, the presence of ions in aquatic matrix can affect these yields. Hence, further photodegradation studies were performed in tap water (Sect. 3.4), and ultimately in synthetic hospital wastewater.

\subsection{Photolysis in Tap Water}

The removal of selected ICM during direct photolysis performed in tap water is presented in Fig. 3. The solution matrix can influence the photochemical reaction, especially inorganic ions present in the matrix. Inhibitory effects may for instance be caused by carbonate $\left(\mathrm{CO}_{3}{ }^{2-}\right)$ and bicarbonate $\left(\mathrm{HCO}_{3}{ }^{-}\right)$anions, which, in combination with the calcium cation, are responsible for the water hardness. The $\mathrm{CO}_{3}{ }^{2-}$ and $\mathrm{HCO}_{3}^{-}$anions can act as
Fig. 2 Photolysis of selected ICM in deionized water $(\mathrm{pH}=7.0)$

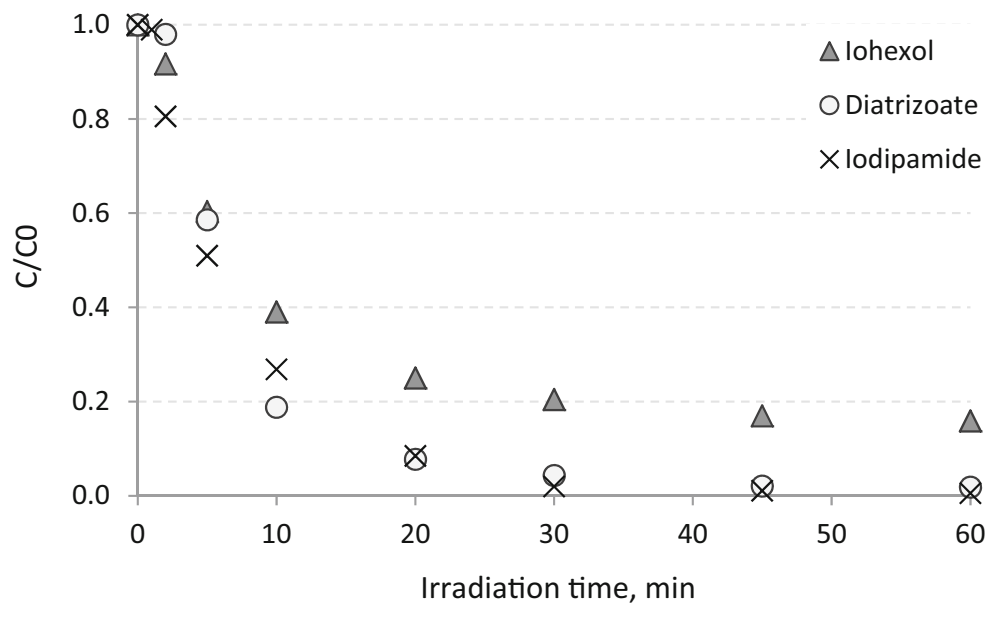


Table 3 Parameters to calculate the quantum yields of selected ICM decay

\begin{tabular}{lllll}
\hline Compounds & $C_{0}\left(\mathrm{~mol} \mathrm{~L}^{-1}\right)$ & $b(\mathrm{~cm})$ & $\varepsilon\left(\mathrm{L} \mathrm{mol}^{-1} \mathrm{~cm}^{-1}\right)$ & $1.71 \times 10^{4}$ \\
\hline Iohexol & $1.35 \times 10^{-5}$ & 1.0 & $1.04 \times 10^{5}$ & $1.52 \times 10^{-8}$ \\
Diatrizoate & $8.11 \times 10^{-6}$ & 1.0 & $5.54 \times 10^{3}$ & $1.98 \times 10^{-8}$ \\
Iodipamide & $8.90 \times 10^{-6}$ & 1.0 & $1.92 \times 10^{-8}$ \\
\hline
\end{tabular}

radical $\cdot \mathrm{OH}$ scavengers and absorb radiation from $\mathrm{UV}$ spectrum (especially from UV-A region) which is required for excitation of the target substance (Arslan et al. 2000). The initial concentrations of ICM spiked into tap water were at the same level as in the case of the deionized water experiment, it means 9.6, 5.1, and $10.3 \mathrm{mg} \mathrm{L}^{-1}$ for iohexol, diatrizoate, and iodipamide, respectively. Among the investigated substances, the efficiency of iohexol removal was (from investigated compounds) the most sensitive to water matrix composition. After $60 \mathrm{~min}$ of direct photolysis performed in tap water, the removal of iohexol was at the level of $72 \%$. This means that decomposition in tap water was reduced by $12 \%$ compared to the efficiency obtained in deionized water. For diatrizoate and iodipamide a significant influence of the matrix on the photolysis efficiency was not observed. After $30 \mathrm{~min}$ of the process, the concentrations of diatrizoate and iodipamide were below LOQ, which means that both substances were removed efficiently.

\subsection{Determination of Optimum Retention (Irradiation)}

Time in UV Reactor

In this study, the optimal retention time of the synthetic hospital wastewater (containing the studied ICM) in the
$\mathrm{UV}$ reactor was determined. The UV reactor was later used as the pretreatment step before the biological treatment in removal of ICM from the hospital wastewater (hybrid system; Sect. 3.4).

The solution of synthetic hospital wastewater spiked with ICM was irradiated for $30 \mathrm{~min}$ (Fig. 4). After $2 \mathrm{~min}$ of UV irradiation, the removal efficiency of iodipamide and iohexol was 7 and $3.5 \%$, respectively, whereas the diatrizoate was already removed in $43 \%$ (Fig. 4). The complete elimination of diatrizoate occurred after $5 \mathrm{~min}$ of the process, whereas the complete elimination of iodipamide was obtained after $10 \mathrm{~min}$. Thirty minutes of the experiment resulted in only $20 \%$ of iohexol decay. For further studies, the optimal media retention time in UV reactor was established as $4 \mathrm{~min}$. Such of value of this parameter caused significant removal of all the investigated compounds, i.e., diatrizoate was removed in $66 \%$, iodipamide in $41 \%$, and iohexol in $13 \%$. Additionally, during such irradiation time no mineralization of organic pollutants was observed - average COD value measured in wastewater before and after UV process remained at the same level (about $1300 \mathrm{mg} \mathrm{O}_{2} \mathrm{~L}^{-1}$ ). The investigations conducted in synthetic wastewater confirmed that iohexol was the least susceptible to UV radiation and that the wastewater
Fig. 3 Photolysis of selected ICM in tap water $(\mathrm{pH}=7.4)$

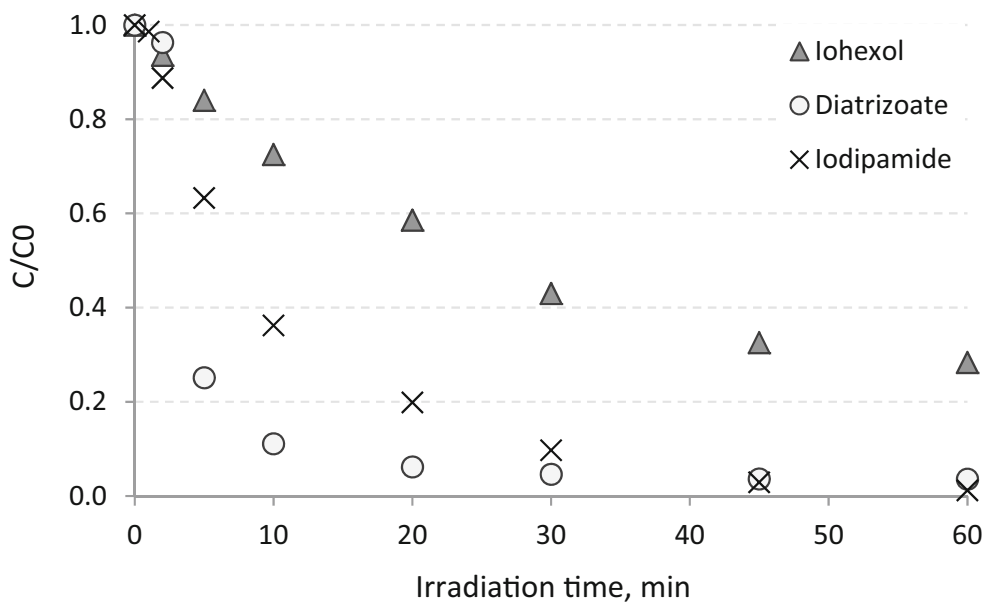


Fig. 4 Determination of retention (irradiation) time of hospital wastewater in $\mathrm{UV}$ reactor $(\mathrm{pH} \approx$ 7.4)

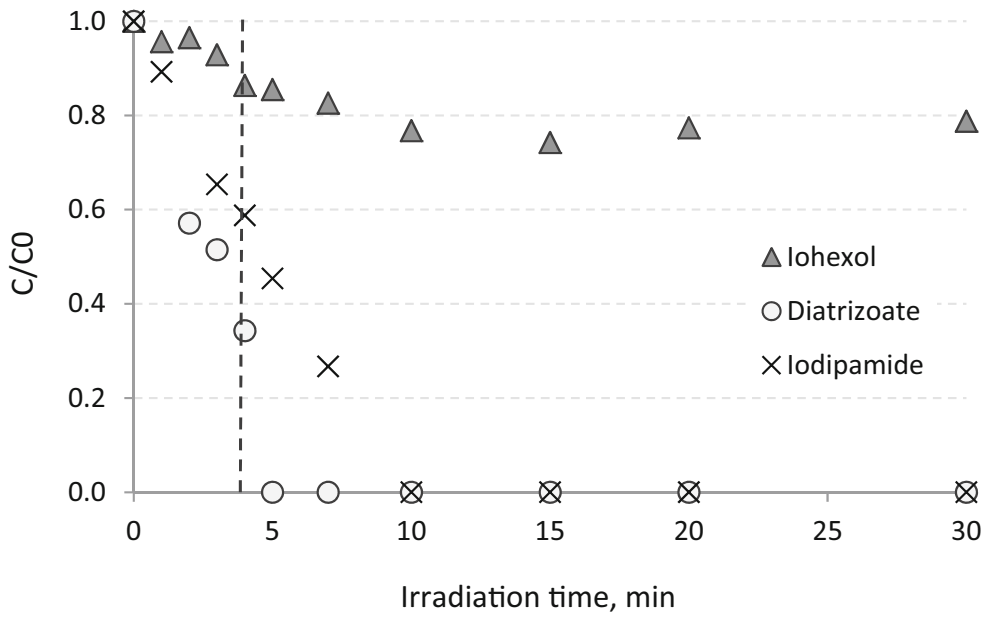

matrix composition (in which the process took place) had a more pronounced adverse effect than in tap water (after 30 min of irradiation $58 \%$ iohexol removal in tap water, compared to only $20 \%$ removal in the synthetic wastewater, Figs. 2 and 3).

\subsection{Determination of Optimum $\mathrm{TiO}_{2}$ Concentration for $\mathrm{UV} / \mathrm{TiO}_{2}$ Process}

Photocatalytic processes with $\mathrm{TiO}_{2}$ addition have been described as an effective method for micropollutant degradation (Doll and Frimmel 2003, 2004; Ternes et al. 2003; Pastrana-Martinez et al. 2012). In order to introduce $\mathrm{TiO}_{2}$ as a pretreatment step before the membrane bioreactors, its optimal dose was determined. Three doses of $\mathrm{TiO}_{2}$ were selected for the investigation: 100,300 , and $500 \mathrm{mg} \mathrm{L}^{-1}$. The first step estimated the ability of ICM adsorption onto the $\mathrm{TiO}_{2}$ surface. The ICM solutions (Sect. 2.3) were mixed with suspensions of $\mathrm{TiO}_{2}$ (at concentrations of 100,300 , and $500 \mathrm{mg} \mathrm{L}^{-1}$ ). Each experiment lasted $10 \mathrm{~min}$, and the results are summarized in Table 4. Iodipamide was the most efficiently removed from the reaction solution as a result of adsorption onto the surface of $\mathrm{TiO}_{2}$. Its removal efficiency was proportional to the applied $\mathrm{TiO}_{2}$ dose and contact time. The maximum iodipamide removal as a result of adsorption onto the $\mathrm{TiO}_{2}$ surface, which is $17 \%$, was observed when the applied dose of $\mathrm{TiO}_{2}$ was equal to $500 \mathrm{mg} \mathrm{L}^{-1}$. In the case of diatrizoate, its removal was insignificant and after $10 \mathrm{~min}$ of the experiment with the highest dose of $\mathrm{TiO}_{2}\left(500 \mathrm{mg} \mathrm{L}^{-1}\right)$ did not exceed the value of $7 \%$. Iohexol removal due to the adsorption process was at the level of $10 \%$, regardless of the applied $\mathrm{TiO}_{2}$ dose. As reported by Doll and Frimmel (2004), the sorption coefficient may be estimated according to Henry equation, when applied to the low concentration range and in the linear part of the sorption data. This approach was used in our

Table 4 Sorption of selected ICM onto $\mathrm{TiO}_{2}$ surface

\begin{tabular}{|c|c|c|c|c|}
\hline \multirow{2}{*}{$\begin{array}{l}\mathrm{TiO}_{2} \text { dose } \\
\left(\mathrm{mg} \mathrm{L}^{-1}\right)\end{array}$} & \multirow{2}{*}{$\begin{array}{l}\text { Time } \\
(\mathrm{min})\end{array}$} & \multicolumn{3}{|c|}{ Relative concentration $C / C_{0}$} \\
\hline & & Iohexol $^{\mathrm{a}}$ & Diatrizoate $^{\mathrm{b}}$ & Iodipamide $^{c}$ \\
\hline \multirow[t]{6}{*}{100} & 0 & 1.00 & 1.00 & 1.00 \\
\hline & 2 & 0.90 & 0.98 & 0.95 \\
\hline & 4 & 0.93 & 0.99 & 0.92 \\
\hline & 6 & 0.94 & 0.99 & 0.92 \\
\hline & 8 & 0.90 & 1.00 & 0.92 \\
\hline & 10 & 0.89 & 0.97 & 0.91 \\
\hline \multirow[t]{6}{*}{300} & 0 & 1.00 & 1.00 & 1.00 \\
\hline & 2 & 0.91 & 0.94 & 0.90 \\
\hline & 4 & 0.00 & 0.95 & 0.92 \\
\hline & 6 & 0.91 & 0.95 & 0.91 \\
\hline & 8 & 0.93 & 0.94 & 0.91 \\
\hline & 10 & 0.89 & 0.93 & 0.86 \\
\hline \multirow[t]{6}{*}{500} & 0 & 1.00 & 1.00 & 1.00 \\
\hline & 2 & 0.99 & 0.94 & 0.91 \\
\hline & 4 & 0.91 & 0.94 & 0.90 \\
\hline & 6 & 0.91 & 0.94 & 0.90 \\
\hline & 8 & 0.90 & 0.94 & 0.88 \\
\hline & 10 & 0.90 & 0.93 & 0.83 \\
\hline
\end{tabular}

${ }^{\mathrm{a}} C_{0}=1.82 \mathrm{mg} \mathrm{L}^{-1}$

${ }^{\mathrm{b}} C_{0}=1.06 \mathrm{mg} \mathrm{L}^{-1}$

${ }^{\mathrm{c}} C_{0}=1.98 \mathrm{mg} \mathrm{L}^{-1}$ 
Fig. 5 Removal of ICMs from hospital wastewater in UV process enhanced by $\mathrm{TiO}_{2}$ in concentration of $100 \mathrm{mg} \mathrm{L}^{-1}$

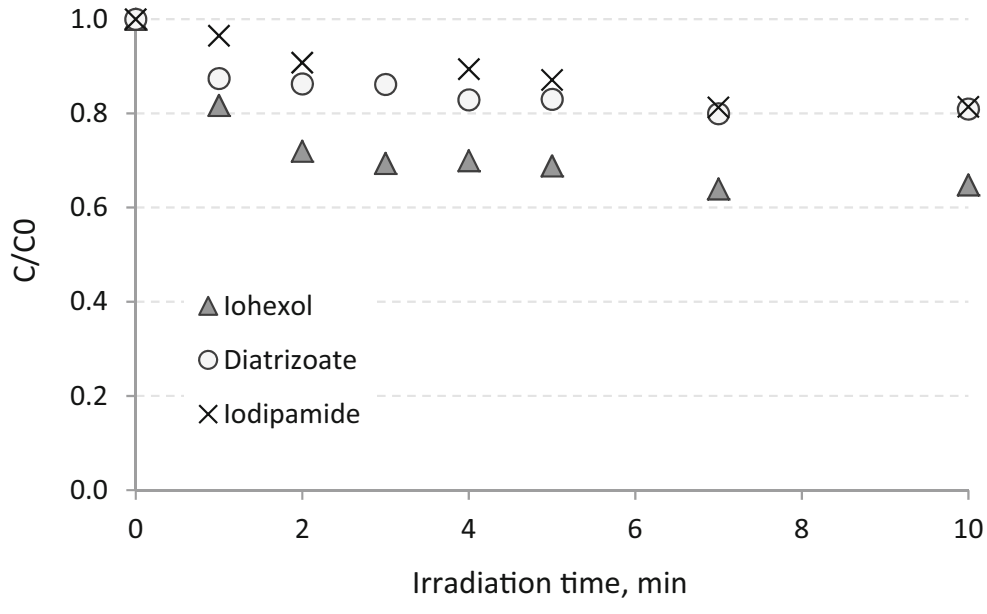

investigation. Thus, the estimated Henry constants, $K_{\mathrm{H}}$ ( $\mathrm{pH}=7.4 ; T \approx 25^{\circ} \mathrm{C}, \mathrm{TiO}_{2}=0.5 \mathrm{~g} \mathrm{~L}^{-1}$ ) for the investigated substances were $0.36,0.29$, and $0.68 \mathrm{mg} \mathrm{g}^{-1}$ for iohexol, diatrizoate, and iodipamide, respectively. It means that from the investigated compounds, iodipamide had the maximum adsorption capacity onto $\mathrm{TiO}_{2}$ surface.

In $\mathrm{UV} / \mathrm{TiO}_{2}$ processes pollutants may be oxidized by both electron holes generated as a result of $\mathrm{TiO}_{2}$ excitation by the UV radiation as well as free radical either at the photocatalyst surface or solution bulk phase (Arslan et al. 2000). During UV/TiO 2 process with the lowest concentration of $\mathrm{TiO}_{2}\left(100 \mathrm{mg} \mathrm{L}{ }^{-1}\right)$, iohexol was removed with the highest efficiency, and after $10 \mathrm{~min}$, it was eliminated in $35 \%$, whereas the diatrizoate and iodipamide decay did not exceed $20 \%$ (Fig. 5). After 4 min of irradiation (Sect. 3.2), iohexol, iodipamide, and diatrizoate were removed from hospital wastewater in
30, 10, and $17 \%$, respectively. In almost all cases, increasing the concentration of $\mathrm{TiO}_{2}$ improved the efficiency of ICM oxidation. After 10 min of irradiation in the presence of $300 \mathrm{mg} \mathrm{TiO} \mathrm{L}^{-1}$, diatrizoate was removed twice as effectively as in the presence of $100 \mathrm{mg}$ $\mathrm{TiO}_{2} \mathrm{~L}^{-1}$ (19 and $41 \%$, respectively) (Fig. 6). Transformation of iodipamide was also more effective-after $10 \mathrm{~min}$ of the processes, the decay of almost $50 \%$ was observed. On the other hand, higher concentration of $\mathrm{TiO}_{2}$ did not increase the efficiency of the iohexol removal, because after $10 \mathrm{~min}$ of the experiment with $300 \mathrm{mg} \mathrm{TiO} \mathrm{L}^{-1}$, similar results as in the experiment with $100 \mathrm{mg} \mathrm{TiO}_{2} \mathrm{~L}^{-1}$ was observed.

The highest removal efficiency for all the investigated ICM was observed in $\mathrm{UV} / \mathrm{TiO}_{2}$ process enhanced by dose of $500 \mathrm{mg} \mathrm{TiO}_{2} \mathrm{~L}^{-1}$ (Fig. 7). Significant ICM decay was observed after $4 \mathrm{~min}$ of the process-for diatrizoate, it was calculated as $43 \%$, for iodipamide
Fig. 6 Removal of ICMs from hospital wastewater in UV process enhanced by $\mathrm{TiO}_{2}$ in concentration of $300 \mathrm{mg} \mathrm{L}^{-1}$

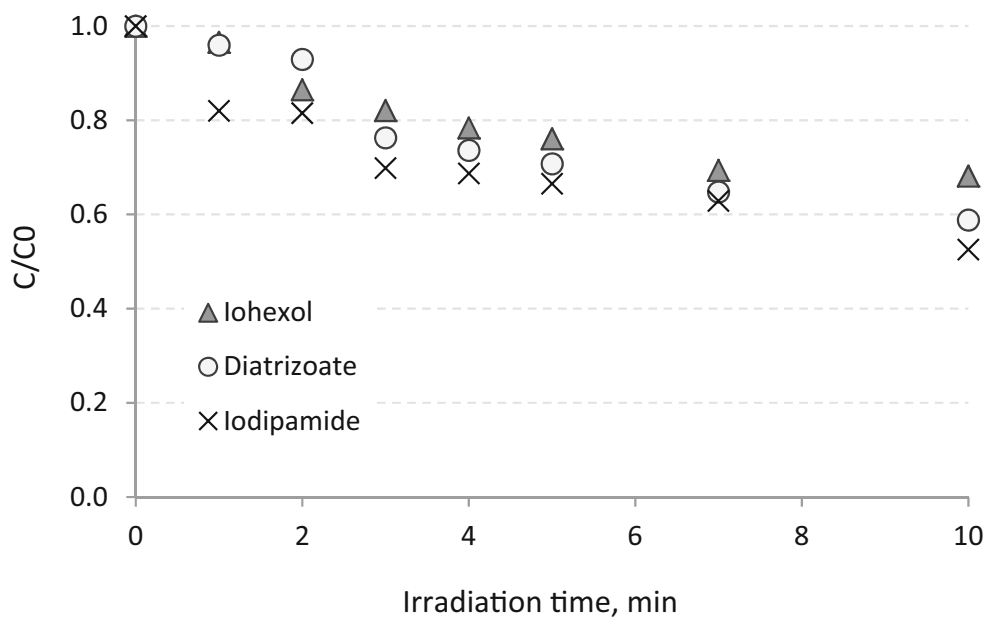


Fig. 7 Removal of ICMs from hospital wastewater in UV process enhanced by $\mathrm{TiO}_{2}$ in concentration of $500 \mathrm{mg} \mathrm{L}^{-1}$

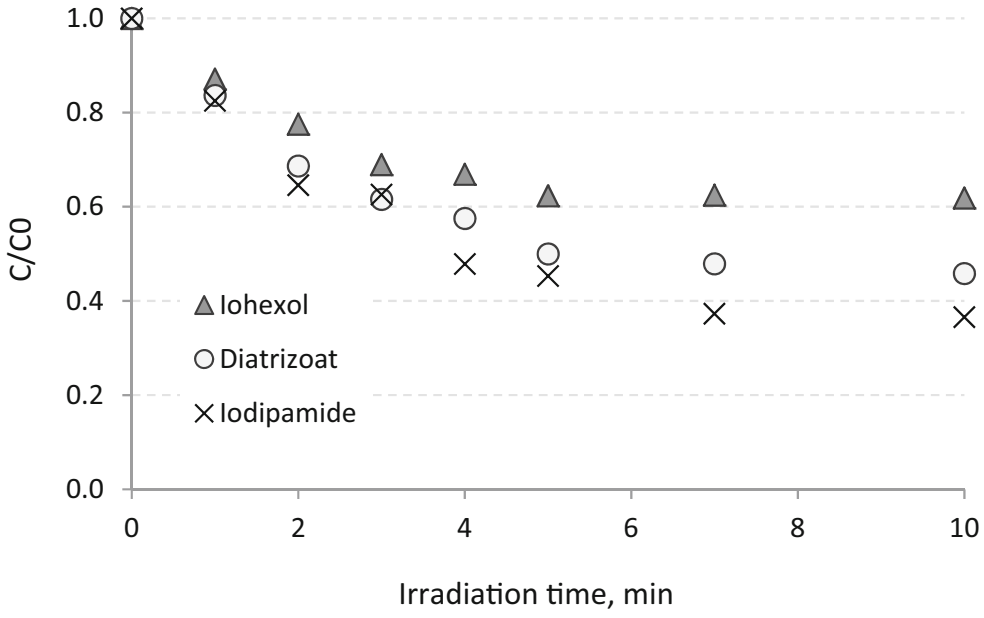

$52 \%$, and for iohexol $33 \%$. After $10 \mathrm{~min}$ of the experiment, more than $50 \%$ of the removal was observed for diatrizoate and iodipamide (54 and $63 \%$, respectively). Iohexol removal (38\%) was at the same level as in the experiments with lower concentration of $\mathrm{TiO}_{2}$. According to the obtained data, iodipamide was the most susceptible to degradation in the $\mathrm{UV} / \mathrm{TiO}_{2}$ process, which was possibly related to its adsorption onto the surface of semiconductor (Doll and Frimmel 2004). Based on the results, it can be also concluded that determining the optimal dose of $\mathrm{TiO}_{2}$ may significantly improve the efficiency of the $\mathrm{UV} / \mathrm{TiO}_{2}$ process.

3.7 ICM Removal from Synthetic Hospital Wastewater in the UV, UV/TiO 2 , and the Hybrid Process

Results below compare the effectiveness of UV and UV/ $\mathrm{TiO}_{2}$ processes with the hybrid installation, at 4-min retention time in the UV reactor and $500 \mathrm{mg} \mathrm{L}^{-1}$ of $\mathrm{TiO}_{2}$ in the UV/TiO 2 process. The hybrid installation combined both photochemical ( $\mathrm{UV}$ or $\mathrm{UV} / \mathrm{TiO}_{2}$ ) and biological processes (MBR), with parameters described in Sects. 3.1-3.3 used in the study. In this part of the study, the UV reactor was operated as plug-flow reactor, contrary to the previous investigations (Sects. 3.2 and 3.3), where it was operated in a recirculation loop. The results on the ICM removal obtained in the preliminary tests (with $\mathrm{UV}$ and $\mathrm{UV} / \mathrm{TiO}_{2}$ only) differed from those obtained in the lab-scale hybrid installation, which means that the different modes of UV reactor operation could influence the removal efficiency of the target substances.

During this experiment, the removal efficiency of diatrizoate was higher in the $\mathrm{UV} / \mathrm{TiO}_{2}$ experiments than in the UV experiments and equaled to 40 and $30 \%$, respectively. In the case of iohexol, $27 \%$ of this compound was removed in the UV process. Addition of $500 \mathrm{mg} \mathrm{TiO} \mathrm{L}^{-1}$ in the $\mathrm{UV} / \mathrm{TiO}_{2}$ process improved the iohexol decay to $38 \%$. No significant differences in removal efficiency of iodipamide during UV and UV// $\mathrm{TiO}_{2}$ processes were observed - the removal efficiency was equal to 29 and $28 \%$, respectively, in UV and UV// $\mathrm{TiO}_{2}$ processes (Fig. 8). The obtained results showed that not in every case the addition of $\mathrm{TiO}_{2}$ to the UV process significantly improved the efficiency of ICM decomposition.
Fig. 8 Comparison of effectiveness of $\mathrm{UV}$ and $\mathrm{UV} / \mathrm{TiO}_{2}$ processes in ICM removal from hospital wastewater (plug-flow UV reactor, irradiation time $=$ $4 \mathrm{~min}$ )

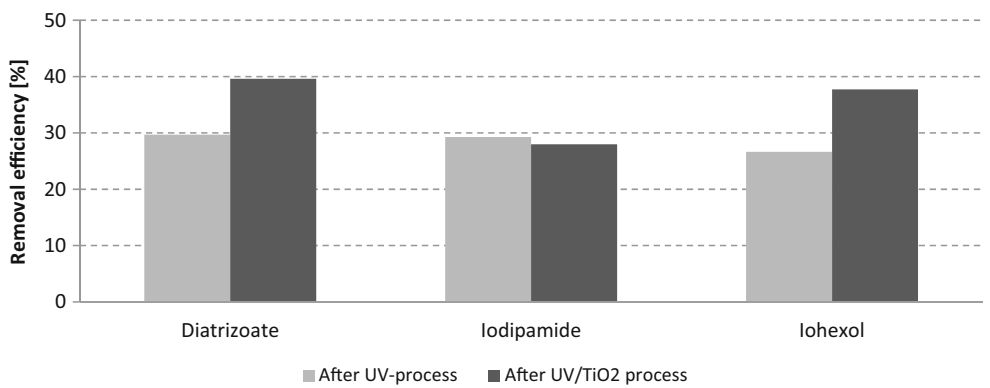


Fig. 9 Comparison of effectiveness of ICM removal from hospital wastewater by means of MBR combined with various types of photochemical pretreatment

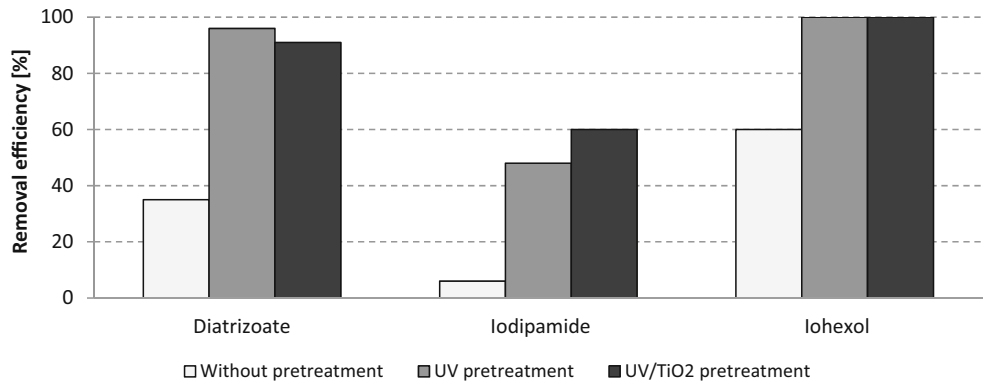

The results from combined photochemical and biological treatment (Fig. 9) show that the use of photochemical pretreatment $\left(\mathrm{UV}\right.$ or $\mathrm{UV} / \mathrm{TiO}_{2}$ ) significantly improved the ICM elimination in the biological treatment step. The removal efficiencies in the biological step without pretreatment equaled to $38 \%$ for diatrizoate, $6 \%$ for iodipamide, and $60 \%$ for iohexol. With pretreatment (regardless whether only UV or UV/ $\mathrm{TiO}_{2}$ were used), more than $90 \%$ elimination of diatrizoate was obtained, while iohexol was removed completely (100\%). For iodipamide, which was practically previously nonbiodegradable, application of the UV radiation as pretreatment before MBR caused its $50 \%$ degradation, while application of the $\mathrm{UV} / \mathrm{TiO}_{2}$ process increased its elimination efficiency to $60 \%$. However, no significant differences in removal efficiency was observed between the UV treatment alone or in combination with the $\mathrm{TiO}_{2}$ process (Fig. 9), indicating that the wastewater matrix played a role in diminishing the $\mathrm{TiO}_{2}$ particles efficiency. In summary, inserting the photochemical processes as a pretreatment step before the biological treatment significantly improved the removal of ICM, compared to the photochemical or to the biological processes alone.

\section{Conclusions}

The experiments showed that elimination of selected ICM from water can be successfully conducted with the photochemical UV and $\mathrm{UV} / \mathrm{TiO}_{2}$ processes. During the experiments, the quantum yields of ICM decay in ideal aqueous solution (deionized water, $\mathrm{pH}=7.0$ ) were established as 0.006, 0.004, and 0.029 for iohexol, diatrizoate, and iodipamide, respectively. This means that iodipamide was the most susceptible to degradation by direct photolysis (UV) induced by the polychromatic irradiation. However, iohexol was the most efficiently degraded in the photochemical $\mathrm{UV} / \mathrm{TiO}_{2}$ process. More importantly, UV and $\mathrm{UV} / \mathrm{TiO}_{2}$ processes were successfully applied as a pretreatment step before a biological wastewater treatment stage (hybrid methods). The photochemical processes disrupted the chemical structure of the studied ICM and this enables them to the further transformation in the biological stage of treatment. Such combination of the photochemical and biological processes significantly enhanced the removal efficiency of the studied ICM from the synthetic hospital wastewater in comparison with the removal efficiency obtained in the biological processes without photochemical pretreatment. It means that the aforementioned processes can be a promising tool for the removal of the iodinated contrast media from the aquatic environment.

Acknowledgments This research was done with financial support by the Polish Ministry of Science and Higher Education, grant nos. N523034 32/1272 and N523 746340.

Open Access This article is distributed under the terms of the Creative Commons Attribution License which permits any use, distribution, and reproduction in any medium, provided the original author(s) and the source are credited.

\section{References}

Arslan, I., Balcioglu, I., Tuhkanen, T., \& Bahnemann, D. (2000). $\mathrm{H} 2 \mathrm{O} 2 / \mathrm{UV}-\mathrm{C}$ and $\mathrm{Fe} 2+/ \mathrm{H} 2 \mathrm{O} 2 / \mathrm{UV}-\mathrm{C}$ versus $\mathrm{TiO} 2 / \mathrm{UV}-\mathrm{A}$ treatment for reactive dye wastewater. Journal of Environmental Engineering and Science, 126, 903-911.

Christiansen, C. (2005). X-ray contrast media-an overview. Toxicology, 209, 185-187.

Daughton, C. G., \& Ternes, T. A. (1999). Pharmaceuticals and personal care products in the environment: agents of subtle change? Environmental Health Perspectives, 107, 907-938.

Doll, T. E., \& Frimmel, F. H. (2003). Fate of pharmaceuticalsphotodegradation by simulated solar UV-light. Chemosphere, 52, 1757-1769.

Doll, T. E., \& Frimmel, F. H. (2004). Kinetic study of photocatalytic degradation of carbamazepine, clofibric acid, iomeprol and iopromide assisted by different $\mathrm{TiO} 2$ materials- 
determination of intermediates and reaction pathways. Water Research, 38, 955-964.

Felis, E., Wiszniowski, J., \& Miksch, K. (2009). Advanced oxidation of diclofenac in various aquatic environments. Archives of Environmental Protection, 35, 15-25.

Felis, E., Ledakowicz, S., \& Miller, J. S. (2011). Degradation of bisphenol a using $\mathrm{UV}$ and $\mathrm{UV} / \mathrm{H} 2 \mathrm{O} 2$ processes. Water Environment Research, 83, 2154-2158.

Haiss, A., \& Kummerer, K. (2006). Biodegradability of the X-ray contrast compound diatrizoic acid, identification of aerobic degradation products and effects against sewage sludge micro-organisms. Chemosphere, 62, 294-302.

Halling-Sorensen, B., Nielsen, S. N., Lanzky, P. F., Ingerslev, F., Lutzhoft, H. C. H., \& Jorgensen, S. E. (1998). Occurrence, fate and effects of pharmaceutical substances in the environment - a review. Chemosphere, 36, 357-394.

Heberer, T. (2002). Occurrence, fate, and removal of pharmaceutical residues in the aquatic environment: a review of recent research data. Toxicology Letters, 131, 5-17.

Huber, M. M., Gobel, A., Joss, A., Hermann, N., Loffler, D., McArdell, C. S., Ried, A., Siegrist, H., Ternes, T. A., \& von Gunten, U. (2005). Oxidation of pharmaceuticals during ozonation of municipal wastewater effluents: a pilot study. Environmental Science and Technology, 39, 4290-4299.

Klavarioti, M., Mantzavinos, D., \& Kassinos, D. (2009). Removal of residual pharmaceuticals from aqueous systems by advanced oxidation processes. Environment International, 35, 402-417.

Knodel, J., Geissen, S. U. J., Broll, J., \& Duennbier, U. (2011). Simulation and source identification of X-ray contrast media in the water cycle of Berlin. Journal of Environmental Management, 92, 2913-2923.

Kummerer, K. (2001). Drugs in the environment: emission of drugs, diagnostic aids and disinfectants into wastewater by hospitals in relation to other sources-a review. Chemosphere, 45, 957-969.

Kummerer, K. (2009). The presence of pharmaceuticals in the environment due to human use-present knowledge and future challenges. Journal of Environmental Management, 90, 2354-2366.

Kwon, M., Yoon, Y., Cho, E., Jung, Y., Lee, B.-C., Paeng, K.-J., \& Kang, J.-W. (2012). Removal of iopromide and degradation characteristics in electron beam irradiation process. Journal of Hazardous Materials, 227, 126-134.

Miksch, K., Cema, G., Corvini, P. F., Felis, E., Sochacki, A., Surmacz-Gorska, J., Wiszniowski, J., \& Zabczynski, S. (2015). R\&D priorities in the field of sustainable remediation and purification of agro-industrial and municipal wastewater. New Biotechnology, 32(1), 128-132.
Miller, J. S., \& Olejnik, D. (2001). Photolysis of polycyclic aromatic hydrocarbons in water. Water Research, 35, 233-243.

Mompelat, S., Le Bot, B., \& Thomas, O. (2009). Occurrence and fate of pharmaceutical products and by-products, from resource to drinking water. Environment International, 35, 803-814.

Ning, B., Graham, N. J. D., \& Lickiss, P. D. (2009). A comparison of ultrasound-based advanced oxidation processes for the removal of X-ray contrast media. Water Science and Technology, 60, 2383-2390.

Pastrana-Martinez, L. M., Morales-Torres, S., Likodimos, V., Figueiredo, J. L., Faria, J. L., Falaras, P., \& Silva, A. M. T. (2012). Advanced nanostructured photocatalysts based on reduced graphene oxide-TiO2 composites for degradation of diphenhydramine pharmaceutical and methyl orange dye. Applied Catalysis B, 123, 241-256.

Perez, S., \& Barcelo, D. (2007). Fate and occurrence of X-ray contrast media in the environment. Analytical and Bioanalytical Chemistry, 387, 1235-1246.

Putschew, A., Wischnack, S., \& Jekel, M. (2000). Occurrence of triiodinated X-ray contrast agents in the aquatic environment. Science of the Total Environment, 255, 129-134.

Schulz, M., Löffler, D., Wagner, M., \& Ternes, T. A. (2008). Transformation of the X-ray contrast medium iopromide in soil and biological wastewater treatment. Environmental Science and Technology, 42, 7207-7217.

Seitz, W., Weber, W. H., Jiang, J.-Q., Lloyd, B. J., Maier, M., Maier, D., \& Schulz, W. (2006). Monitoring of iodinated Xray contrast media in surface water. Chemosphere, 64, 13181324.

Steger-Hartmann, T., Lange, R., \& Schweinfurth, H. (1999). Environmental risk assessment for the widely used iodinated X-ray contrast agent iopromide (Ultravist). Ecotoxicology and Environmental Safety, 42, 274-281.

Steger-Hartmann, T., Lange, R., Schweinfurth, H., Tschampel, M., \& Rehmann, I. (2002). Investigations into the environmental fate and effects of iopromide (Ultravist), a widely used iodinated X-ray contrast medium. Water Research, 36, 266274.

Ternes, T. A., \& Hirsch, R. (2000). Occurrence and behavior of Xray contrast media in sewage facilities and the aquatic environment. Environmental Science and Technology, 34, 27412748.

Ternes, T. A., Stuber, J., Herrmann, N., McDowell, D., Ried, A., Kampmann, M., \& Teiser, B. (2003). Ozonation: a tool for removal of pharmaceuticals, contrast media and musk fragrances from wastewater? Water Research, 37, 1976-1982. 